\title{
A Robust Capon Beamformer against Uncertainty of Nominal Steering Vector
}

\author{
Zhu Liang $\mathrm{Yu}^{1}$ and Meng Hwa Er ${ }^{2}$ \\ ${ }^{1}$ Center for Signal Processing, Nanyang Technological University, Singapore 639798 \\ ${ }^{2}$ School of Electrical and Electronic Engineering, Nanyang Technological University, Singapore 639798
}

Received 21 April 2005; Revised 19 October 2005; Accepted 21 October 2005

Recommended for Publication by Fulvio Gini

A robust Capon beamformer (RCB) against the uncertainty of nominal array steering vector (ASV) is formulated in this paper. The $\mathrm{RCB}$, which can be categorized as diagonal loading approach, is obtained by maximizing the output power of the standard Capon beamformer (SCB) subject to an uncertainty constraint on the nominal ASV. The bound of its output signal-to-interference-plusnoise ratio (SINR) is also derived. Simulation results show that the proposed RCB is robust to arbitrary ASV error within the uncertainty set.

Copyright ( 2006 Hindawi Publishing Corporation. All rights reserved.

\section{INTRODUCTION}

Adaptive array has been studied for some decades as an attractive solution to signal detection and estimation in harsh environments. It is widely used in wireless communications, microphone array processing, radar, sonar and medical imaging, and so forth. A well-studied adaptive beamformer, for example, the Capon beamformer [1], has high performance in interference suppression provided that the array steering vector (ASV) corresponding to the signal of interest (SOI) is known accurately.

When adaptive arrays are used in practical applications, some of the underlying assumptions on the environment, sources, and sensor array can be violated. Consequently, there is mismatch between the nominal and actual ASVs. Common array imperfections causing ASV mismatch include steering direction error $[2,3]$, array calibration error [4], near-far field problem [5], multipath or reverberation effects [6], and so forth. Since ASV mismatch gives rise to target signal cancellation in adaptive beamformer, robust beamforming is required in practical applications.

Some robust adaptive beamformers have been proposed to avoid performance degradation due to array imperfections (see $[7,8]$ and references therein). However, most of these methods deal with steering direction error only. When ASV mismatch is caused by array perturbation, array manifold mismodeling, or wavefront distortion, these methods cannot achieve sufficient improvement on robustness [9].
If ASV can be modeled as a vector function of some parameters, like steering direction error [10] and time-delay error or general-phase-error (GPE) between sensors [11, 12], robust beamformer can be constructed by maximizing the output power of the standard Capon beamformer (SCB) to those parameters in their feasible ranges. Efficient gradient descent-based method [13] can be derived to find the optimal parameters. With these estimated optimal parameters, the error in ASV can be compensated. The signal cancellation effect in the output is then reduced.

In this paper, we further extend the idea used in [10-12] to design an adaptive array robust to arbitrary ASV error. Since the output power of the SCB is a function of the assumed ASV, in this paper, we maximize the output power of the SCB with respect to all feasible ASVs instead of those parameters of the ASV in [10-12]. Although nonzero scaling of ASV does not change the output signal-to-interference-plusnoise ratio (SINR) of the SCB, it introduces an arbitrary scale in the output power. To eliminate this ambiguity of output power, we assume that the ASV has unit norm. If there is no other constraint on the ASV, the design of the array processor can be simplified to a principal (minor) component analysis problem (PCA/MCA) [14]. Nevertheless, when the target signal is not the dominant one, such array processor may wrongly suppress the target signal and retrieve interference as the output signal. To solve this problem, we introduce an additional uncertainty constraint on the ASV. This uncertainty constraint of the ASV is also used in some robust 
methods [15-18]. It assumes that the feasible ASV is in an ellipsoid whose center is the nominal ASV. With this uncertainty constraint, the designed Capon beamformer is robust to arbitrary ASV error even with the existence of strong interferences. We also derive the robust beamformer using a new idea by maximizing the output power of the SCB; the derived RCB has similar mathematical form as the beamformer in [18]. Theoretical analysis shows that the proposed RCB can be generalized as a diagonal loading approach. The diagonal loading factor is calculated from the constraint equation. In this paper, we derive the optimal output SINR of the proposed RCB. Unfortunately, the calculated diagonal loading factor for the proposed RCB is not in the theoretical range of the optimal factor, meaning that the proposed RCB cannot achieve the optimal output SINR. However, numerical experiments show that the RCB demonstrates outstanding robustness to ASV error and has relatively high output SINR.

This paper is organized as follows. The derivation of RCB and the performance analysis are given in Sections 2 and 3, respectively. Some numerical results are shown in Section 4 to evaluate the performance of the proposed RCB. In Section 5, a brief conclusion is given.

\section{PROPOSED METHOD}

Assume that the signals from $K$ uncorrelated sources imping on an array comprising $M$ isotropic sensors. The power and the ASV of the SOI are $\left\{\sigma_{s}^{2}, \mathbf{s}_{0}\right\}$ and those of the interferences are $\left\{\sigma_{k}^{2}, s_{k}\right\}, k \geq 1$. The theoretical covariance matrix of the array snapshot is given by

$$
\mathbf{R}=\sigma_{s}^{2} \mathbf{s}_{0} \mathbf{s}_{0}^{H}+\sum_{k=1}^{K-1} \sigma_{k}^{2} \mathbf{s}_{k} \mathbf{s}_{k}^{H}+\mathbf{Q}
$$

where $M \times M$ matrix $\mathbf{Q}$ is the covariance matrix of nondirectional noise. It usually has full rank. In practical applications, $\mathbf{R}$ is replaced by the sample covariance matrix $\hat{\mathbf{R}}$,

$$
\widehat{\mathbf{R}}=\frac{1}{N} \sum_{n=1}^{N} \mathbf{x}_{n} \mathbf{x}_{n}^{H},
$$

where $N$ denotes the number of the snapshots and $\mathbf{x}_{n}$ represents the $n$th snapshot.

If the steering vector $\mathbf{s}_{0}$ of the SOI is known, the Capon beamformer is formulated as a linearly constrained quadratic optimization problem. It minimizes the output power with the constraint that the gain of the signal from the direction of interest is unity, which can be expressed as

$$
\min _{\mathbf{w}} \mathbf{w}^{H} \hat{\mathbf{R}} \mathbf{w} \quad \text { s.t. } \mathbf{s}_{0}^{H} \mathbf{w}=1 \text {, }
$$

where $\mathbf{w}$ is the weight vector of the beamformer. The optimal weight $\mathbf{w}_{0}$ and the output power $\hat{\sigma}_{s}^{2}$ of the SOI are

$$
\mathbf{w}_{0}=\frac{\hat{\mathbf{R}}^{-1} \mathbf{s}_{0}}{\mathbf{s}_{0}^{H} \hat{\mathbf{R}}^{-1} \mathbf{s}_{0}}, \quad \hat{\sigma}_{s}^{2}=\frac{1}{\mathbf{s}_{0}^{H} \hat{\mathbf{R}}^{-1} \mathbf{s}_{0}} .
$$

It is known that nonzero scaling of $\mathbf{s}_{0}$ does not change the output SINR of the adaptive beamformer. However, it changes the estimated output power in (4). Without loss of generality, we assume that $\mathbf{s}_{0}$ has unit norm to eliminate the ambiguity in the output power.

In practical applications, the ASV $\mathbf{s}_{0}$ is always unknown or known but with some error. If $\mathbf{s}_{0}$ deviates from the true one, target signal cancellation is inevitable. This results in decrease of output power in (4). A solution to this problem is to search for an optimal ASV s, which results in maximal output power $\hat{\sigma}_{s}^{2}[10-12]$. Therefore, the robust beamformer can be formulated as

$$
\max _{\mathbf{s}} \min _{\mathbf{w}} \mathbf{w}^{H} \hat{\mathbf{R}} \mathbf{w} \quad \text { s.t. } \mathbf{s}^{H} \mathbf{w}=1,\|\mathbf{s}\|^{2}=1,
$$

where $\|\cdot\|^{2}$ denotes the Euclidian norm.

This optimization problem can be solved in two steps. First, we fix $s$ and search for the minimal output power. Then we search for the maximal value of the minimal output power to all the feasible s. For any given $\mathbf{s}$, the output power of the SCB is expressed in (4). Since $\mathbf{s}^{H} \hat{\mathbf{R}}^{-1} \mathbf{s}$ is a scale, maximizing $1 / \mathbf{s}^{H} \hat{\mathbf{R}}^{-1} \mathbf{s}$ is equivalent to minimizing $\mathbf{s}^{H} \hat{\mathbf{R}}^{-1} \mathbf{s}$. The optimization in (5) is simplified to

$$
\min _{\mathbf{s}} \mathbf{s}^{H} \widehat{\mathbf{R}}^{-1} \mathbf{s} \quad \text { s.t. }\|\mathbf{s}\|^{2}=1 \text {, }
$$

which becomes a principal (minor) component analysis problem [14]. The optimal $\widehat{s}$ is the eigenvector corresponding to the largest eigenvalue of $\hat{\mathbf{R}}$.

However, if the target signal is not the dominant one, this method leads to a wrong solution. Therefore, additional constraint must be incorporated in the optimization problem (5). In many cases, $\mathbf{s}_{0}$ is assumed to be known but with some error. For example, $\mathbf{s}_{0}$ belongs to the following uncertainty set [15-18]:

$$
\mathbf{s}_{0} \in\left\{\mathbf{s} \mid\left\|\mathbf{s}-\overline{\mathbf{s}}_{0}\right\|^{2} \leq \epsilon\right\}
$$

where $\overline{\mathbf{s}}_{0}$ is the nominal ASV with unit norm.

With the uncertainty set of ASV (7), the robust beamformer is constructed by maximizing the output power of the SCB when an imprecise knowledge of its steering vector $\mathbf{s}_{0}$ is available:

$$
\max _{\mathbf{s}} \min _{\mathbf{w}} \mathbf{w}^{H} \hat{\mathbf{R}} \mathbf{w} \quad \text { s.t. } \mathbf{s}^{H} \mathbf{w}=1,\|\mathbf{s}\|^{2}=1,\left\|\mathbf{s}-\overline{\mathbf{s}}_{0}\right\|^{2} \leq \epsilon .
$$

This is equivalent to

$$
\min _{\mathbf{s}} \mathbf{s}^{H} \widehat{\mathbf{R}}^{-1} \mathbf{s} \quad \text { s.t. }\|\mathbf{s}\|^{2}=1, \mathbf{s}^{H} \overline{\mathbf{s}}_{0}+\overline{\mathbf{s}}_{0}^{H} \mathbf{s} \geq 2-\epsilon .
$$

The optimization problem (9) has analogous mathematical expression as that in [16-18] and it can be solved by the Lagrange multiplier methodology [13]. Compare (8) and (9) with (36) in [18]; the difference is the norm of the ASV s. Hence, the solution of (8) can refer to [18]. The optimal solution $\widehat{\mathbf{s}}$ is given by

$$
\widehat{\mathbf{s}}=-\widehat{g}_{2}\left(\widehat{\mathbf{R}}^{-1}+\widehat{g}_{1} \mathbf{I}\right)^{-1} \overline{\mathbf{s}}_{0},
$$

where $\widehat{g}_{1}$ and $\widehat{g}_{2}$ are the estimated Lagrange multipliers. Since the nonzero scale $\hat{g}_{2}$ does not influence the output SINR of 
beamformer, it can be ignored in the analysis of output SINR. It can be proved that $\widehat{g}_{1} \in\left(-1 / \lambda_{1},+\infty\right)$ using similar derivation in [18], where $\lambda_{1}$ is the largest eigenvalue of the covariance matrix $\hat{\mathbf{R}}$. The corresponding optimal weight of the beamformer is given by

$$
\mathbf{w}_{0}=\frac{\widehat{\mathbf{R}}^{-1} \widehat{\mathbf{s}}}{\widehat{\mathbf{s}}^{H} \hat{\mathbf{R}}^{-1} \widehat{\mathbf{s}}},
$$

and the estimate of the signal power $\hat{\sigma}_{s}^{2}$ and the output SINR $\rho$ are given by

$$
\hat{\sigma}_{s}^{2}=\frac{1}{\widehat{\mathbf{s}}^{H} \hat{\mathbf{R}}^{-1} \widehat{\mathbf{s}}}, \quad \rho=\frac{\mathbf{w}_{0}^{H} \hat{\mathbf{R}}_{s} \mathbf{w}_{0}}{\mathbf{w}_{0}^{H}\left(\widehat{\mathbf{R}}_{i}+\widehat{\mathbf{R}}_{n}\right) \mathbf{w}_{0}},
$$

where $\hat{\mathbf{R}}_{s}, \hat{\mathbf{R}}_{i}$, and $\hat{\mathbf{R}}_{n}$ are the covariance matrices of the target signal, interference, and nondirectional noise, respectively.

\section{PERFORMANCE ANALYSIS}

In this section, the bound of the output SINR of the proposed beamformer is derived. A complete performance analysis of the SINR under general array imperfections represents a formidable analytical task. In this paper, we assume that the array processor only has steering vector error. The theoretical covariance matrix is used in the analysis. In such case, the performance degradation of the Capon beamformer is caused by the error in the nominal ASV. The output SINR of the Capon beamformer is given in Lemma 1.

Lemma 1. Assume that the covariance matrices of the SOI and the interference/noise are $\mathbf{R}_{s}$ and $\mathbf{R}_{n}$, respectively. The covariance matrix of array snapshot is $\mathbf{R}=\mathbf{R}_{s}+\mathbf{R}_{n}$. When the nominal $A S V$ is given as $\mathbf{s}$, and the true $A S V$ is given by $\mathbf{s}_{0}$, the output SINR $\rho$ of the Capon beamformer is given by

$$
\rho=\frac{\rho_{o} \cos ^{2}(\theta)}{1+\sin ^{2}(\theta) \rho_{o}\left(\rho_{o}+2\right)},
$$

where $\theta$ is the angle between vector $\mathbf{s}$ and $\mathbf{s}_{0}$, and $\rho_{o}$ is the output SINR of the Capon beamformer when accurate ASV is known, and

$$
\begin{aligned}
\cos ^{2}(\theta) & =\frac{\left|\mathbf{s}_{0}^{H} \mathbf{R}_{n}^{-1} \mathbf{s}\right|^{2}}{\left\|\mathbf{s}_{0}\right\|_{\mathbf{R}}^{2}\|\mathbf{s}\|_{\mathbf{R}}^{2}}, \\
\rho_{o} & =\sigma_{s}^{2} \mathbf{s}_{0}^{H} \mathbf{R}_{n}^{-1} \mathbf{s}_{0}=\sigma_{s}^{2}\left\|\mathbf{s}_{0}\right\|_{\mathbf{R}}^{2},
\end{aligned}
$$

where $\|\mathbf{x}\|_{\mathbf{R}}^{2} \triangleq \mathbf{x}^{H} \mathbf{R}_{n}^{-1} \mathbf{x}$ is the extended vector norm $\left(\mathbf{R}_{n}\right.$ is a positive matrix); $\sigma_{s}^{2}$ is the power of the SOI. If $\mathbf{R}_{n}=\sigma_{n}^{2} \mathbf{I}$, the extended vector norm $\|\cdot\|_{\mathrm{R}}$ can be replaced by the Euclidian norm, and

$$
\cos ^{2}(\theta)=\frac{\left|\mathbf{s}_{0}^{H} \mathbf{s}\right|^{2}}{\left\|\mathbf{s}_{0}\right\|^{2}\|\mathbf{s}\|^{2}}, \quad \rho_{\mathrm{opt}}=\frac{\sigma_{s}^{2}}{\sigma_{n}^{2}}\left\|\mathbf{s}_{0}\right\|^{2} .
$$

Proof. Refer to [19].

Lemma 1 indicates that the output SINR of the Capon beamformer is determined by the angle between the nominal and the true ASVs. Moreover, it is easy to find that the output SINR $\rho$ is a monotonically increasing function of $\cos ^{2}(\theta)$. From (10) and (11), we find that the proposed RCB has similar mathematical form as that of the Capon beamformer except that the nominal vector $\overline{\mathbf{s}}_{0}$ is replaced by the estimated one $\widehat{\mathbf{s}}$. Therefore, the performance of the proposed RCB can be analyzed via the angle between $\widehat{\boldsymbol{s}}$ and $\mathbf{s}_{0}$. Herein, the bound of output SINR of the proposed RCB is derived in Lemma 2.

Lemma 2. Assume that the covariance matrix of the interference/noise is $\mathbf{R}_{n}$ and its eigendecomposition is

$$
\mathbf{R}_{n}=\left[\begin{array}{ll}
\mathbf{U}_{i} & \mathbf{U}_{n}
\end{array}\right]\left[\begin{array}{cc}
\Sigma_{i} & \mathbf{0} \\
\mathbf{0} & \Sigma_{n}
\end{array}\right]\left[\begin{array}{ll}
\mathbf{U}_{i} & \mathbf{U}_{n}
\end{array}\right],
$$

where $\mathbf{U}_{i}$ and $\mathbf{U}_{n}$ are the eigenvector matrices which span the interference and noise subspaces, respectively. The diagonal matrices $\Sigma_{i}=\operatorname{diag}\left\{\lambda_{1}, \ldots, \lambda_{K}\right\}$ and $\Sigma_{n}=\sigma_{n}^{2} \mathbf{I}$ are the corresponding eigenvalue matrices. If $\lambda_{i} \gg \sigma_{n}^{2}, i=1, \ldots, K$, the upper bound $\rho_{u}$ of the output SINR is

$$
\rho_{u}=\frac{\sigma_{s}^{2}\left\|P_{\mathbf{U}_{n}} \mathbf{s}_{0}\right\|^{2}}{\sigma_{n}^{2}},
$$

which is achieved when

$$
g_{1}=\frac{-1}{\sigma_{n}^{2}+\sigma_{s}^{2}\left\|P_{\mathbf{U}_{n}} \mathbf{s}_{0}\right\|^{2}},
$$

provided that $\mathbf{s}_{0}^{H} P_{\mathbf{U}_{n}} \overline{\mathbf{s}} \neq 0$. The matrix $P_{\mathbf{U}_{n}}=\mathbf{U}_{n} \mathbf{U}_{n}^{H}$ is the projection matrix to the subspace spanned by $\mathbf{U}_{n}$. The power and $A S V$ of the SOI are $\sigma_{s}^{2}$ and $\mathbf{s}_{0}$, respectively.

Proof. Refer to the appendix.

Lemma 2 indicates that the optimal output SINR of the RCB is achievable with negative diagonal loading factor. Since $\lambda_{1} \geq \sigma_{n}^{2}+\sigma_{s}^{2}\left\|\mathbf{s}_{0}\right\|^{2} \geq \sigma_{n}^{2}+\sigma_{s}^{2}\left\|P_{\mathbf{U}_{n}} \mathbf{s}_{0}\right\|^{2}$, the optimal value of $g_{1}$ is not in the range $\left(-1 / \lambda_{1}, \infty\right)$ of the solution for the proposed RCB. Nevertheless, the simulation results in the next section will show that the proposed RCB still has high output SINR.

\section{NUMERICAL STUDY}

In this section, some numerical simulations were carried out to evaluate the performance of the proposed RCB. A uniform linear array containing eight sensors with half-wavelength spacing is used to estimate the power of the SOI in the presence of strong interferences as well as uncertainty in the ASV. There are two kinds of uncertainty under consideration. One is the well-studied steering direction error, the other is arbitrary ASV error. In the simulations, the array steering direction error $\Delta$ is assumed to be $3^{\circ}$. The arbitrary ASV error is generated as random zero-mean complex Gaussian vector with norm 0.4. The standard Capon beamformer (SCB) and the generalized-phase-error-based beamformer (GPEB) [11] are included for the purpose of performance comparison.

In the simulations, the estimate of signal power and SINR were the average of 200 Monte Carlo experiments. The 


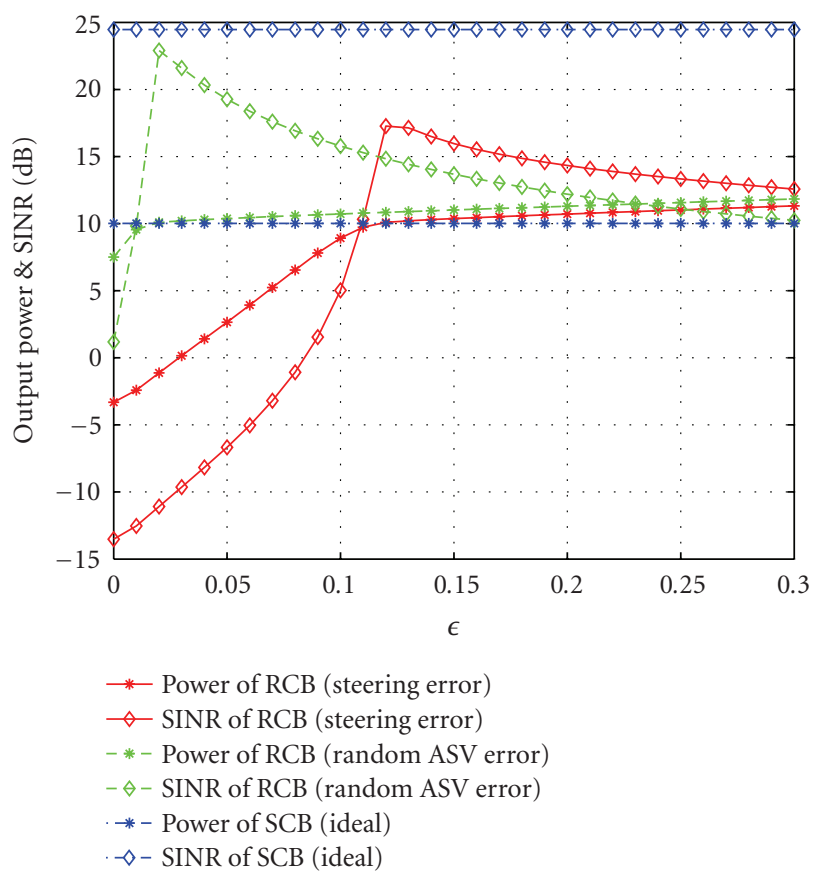

FIgURE 1: Output power and SINR versus the uncertainty level $\epsilon$ (configuration 1).

nondirectional noise is a spatially white Gaussian noise whose power is $-10 \mathrm{~dB}$. The powers and DOAs of the two interferences are $\left(\sigma_{1}^{2}=20 \mathrm{~dB}, \theta_{1}=60^{\circ}\right)$ and $\left(\sigma_{2}^{2}=20 \mathrm{~dB}\right.$, $\theta_{2}=80^{\circ}$ ), respectively. The assumed direction of arrival (DOA) of the SOI is $\theta_{0}=0^{\circ}$. To show the performance of the RCB under different input SINR, two configurations of the SOI are used. In configuration 1, the SOI, which is not the dominant signal, has power $\sigma_{0}^{2}=10 \mathrm{~dB}$. In configuration 2 , the SOI is assumed to be the dominant signal with power $\sigma_{0}^{2}=30 \mathrm{~dB}$.

In the first simulation, the output power and SINR of the RCB versus $\epsilon$ are studied. The results shown in Figure 1 are obtained with configuration 1 . The ideal output power and SINR of the SCB with known ASV are also shown. For any kind of array imperfections, the output SINR of the RCB has a peak value. This can be explained as follows. If small $\epsilon$ is used, the uncertainty constraint does not include the true ASV so that the output SINR is low. When $\epsilon$ is larger than the optimal one, some signal components of the interferences are included in the output signal, resulting in the increase of output power and the decrease of the output SINR, as shown in Figure 1. As we discussed in Section 2, when $\epsilon$ is large enough, the uncertainty constraint is inactive during optimization. In such case, the output power maximization results in target signal cancellation. When correct $\epsilon$ is used, the output of the RCB has highest SINR. However, its output SINR is lower than the ideal one. If the SOI is the dominant signal (configuration 2), the results shown in Figure 2 are different from those shown in Figure 1. When $\epsilon$ is greater than a certain value, the output SINR of the RCB remains constant. The reason is that the optimization problem is simplified as PCA problem in such case. The performance does not change

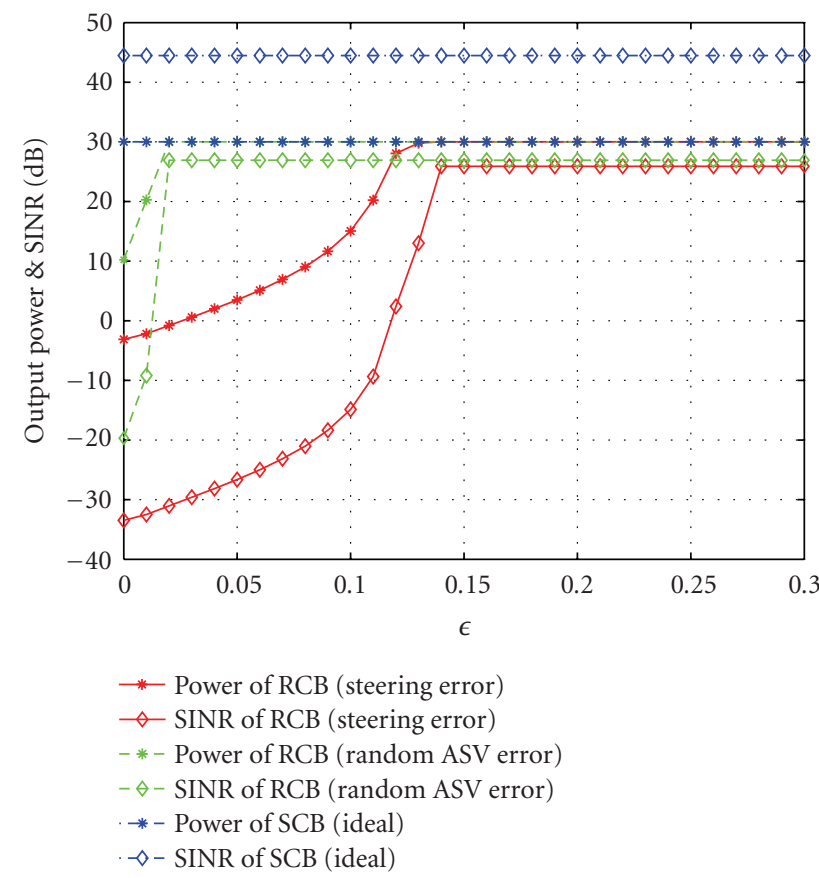

FIgure 2: Output power and SINR versus the uncertainty level $\epsilon$ (configuration 2).

with the increase of $\epsilon$. From the results shown in Figures 1 and 2, we find that the selection of $\epsilon$ is important, especially when the SOI is not the dominant signal. In practical application, $\epsilon_{\mathrm{opt}}$ can be selected as

$$
\epsilon_{\mathrm{opt}}=\min _{\phi}\left\|\mathbf{s}_{0}-e^{-j \phi} \mathbf{s}\right\|^{2},
$$

where $\boldsymbol{s}$ is the ASV with error as discussed in [18].

In the next simulation, we evaluate the performance of the RCB versus the number of sensors. The two curves shown in Figure 3 are obtained using configurations 1 and 2, respectively. The performance of the RCB increases with the number of sensors for both configurations. However, whatever the configuration of signals, the performance of RCB does not change significantly when the number of sensors is larger than a certain value. The reason is that for a given configuration, a certain degree of freedom (DOF) is necessary for interference suppression. Extra DOFs cannot improve the output SINR significantly. On the contrary, it causes target signal cancellation when there are array imperfections [20-22]. This is also the motivation of the partially adaptive beamformer $[21,22]$. Another property is that the RCB has higher SINR improvement when the input SINR is low.

The covariance matrix in the simulation is estimated with limited number of snapshots. It is well known that the covariance matrix estimated using sample averaging method asymptotically approaches the true one. In the case where only a small number of snapshots are available, the estimated error in covariance matrix also affects the performance of beamformer. The results shown in Figure 4 indicate that the output power of the RCB is close to the true one although the number of snapshots is small. With increasing number 


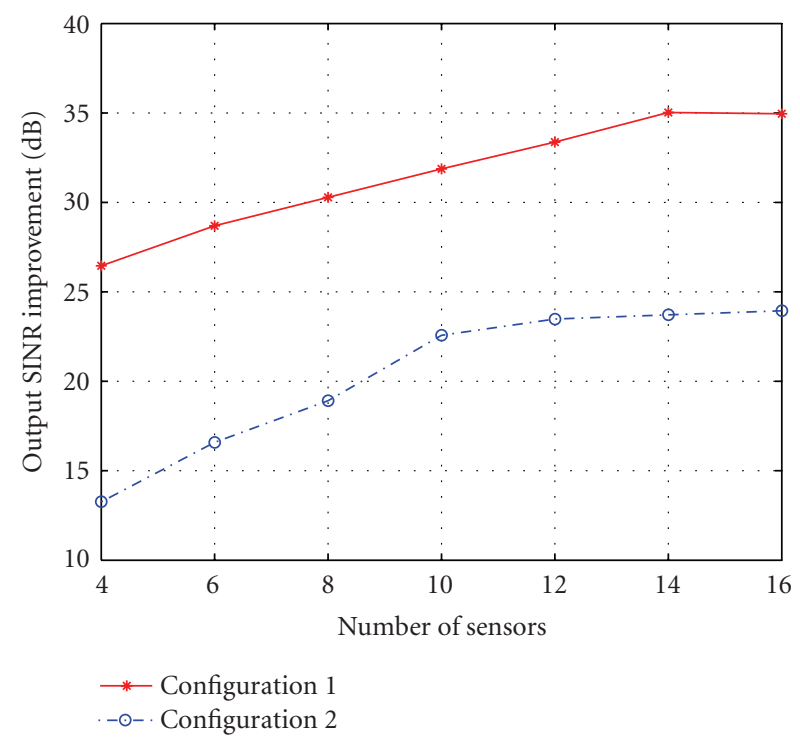

FIGURE 3: Output SINR improvement versus the number of sensors (configuration 1: the SOI is not a dominant signal; configuration 2: the SOI is a dominant signal).

of snapshots, the output SINR is improved for the proposed RCB. However, for the SCB, due to steering direction error, the target signal is cancelled and the output SINR remains at low level. Similar conclusion can be obtained from the results shown in Figure 5, which are obtained with random ASV error.

Compare the performance of the RCB and GPEB shown in Figure 4. It is clear that the GPEB has higher output SINR than that of the RCB when the covariance matrix is estimated with large number of snapshots. The reason is that, when the array imperfection can be modeled as GPE, the GPEB can achieve the same output SINR as the ideal SCB [11]. However, when the covariance matrix is estimated with small number of snapshots, the performance of the GPEB degrades, while the RCB still has higher performance than that of the GPEB. When the array has random ASV error, the results shown in Figure 5 indicate that the performance of the GPEB is poor because the model of the array imperfection used in GPEB is violated. These simulations demonstrate that the RCB can deal with more kinds of array imperfections.

In the next experiment, the power estimates of the signals at different directions are evaluated when the array has arbitrary ASV error. The covariance matrix is estimated from 100 snapshots. The direction and power of the five sources are $\left(-55^{\circ}, 10 \mathrm{~dB}\right),\left(-25^{\circ}, 20 \mathrm{~dB}\right),\left(0^{\circ}, 10 \mathrm{~dB}\right)$, $\left(20^{\circ}, 20 \mathrm{~dB}\right)$, and $\left(50^{\circ}, 20 \mathrm{~dB}\right)$, respectively. With the existence of ASV error, the serious target signal cancellation effect on the SCB gives rise to large error in the estimated output power. On the other hand, the proposed RCB does not suffer from target signal cancellation. The simulation results in Figure 6 show that the proposed RCB gives estimates

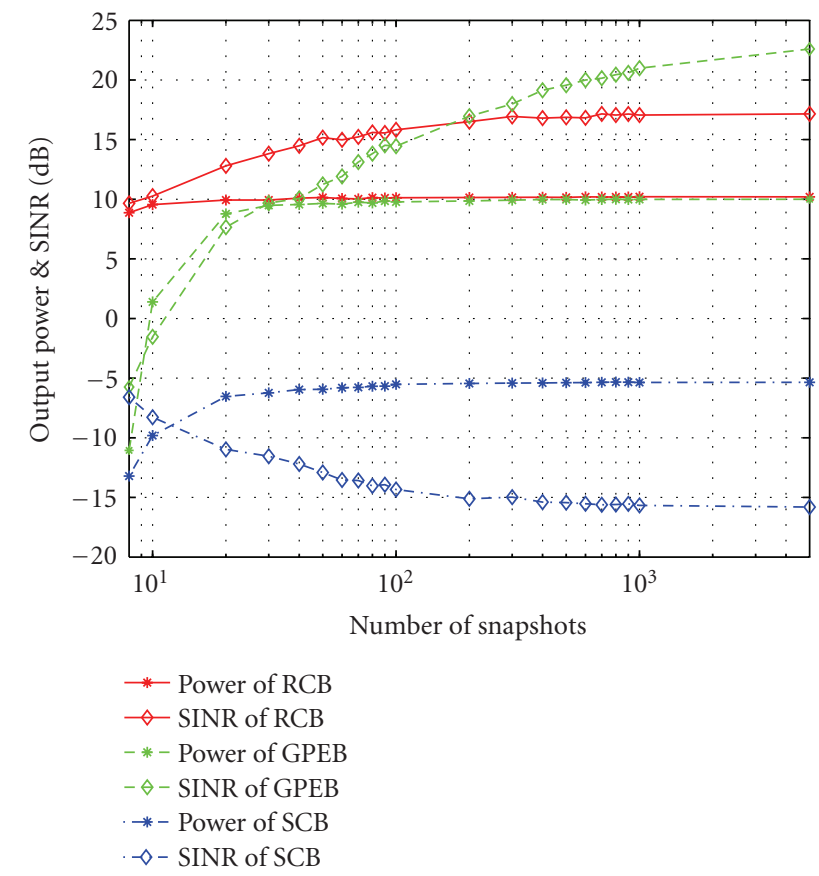

FIGURE 4: Output power and SINR versus the number of snapshots with steering error $(\epsilon=0.13)$.

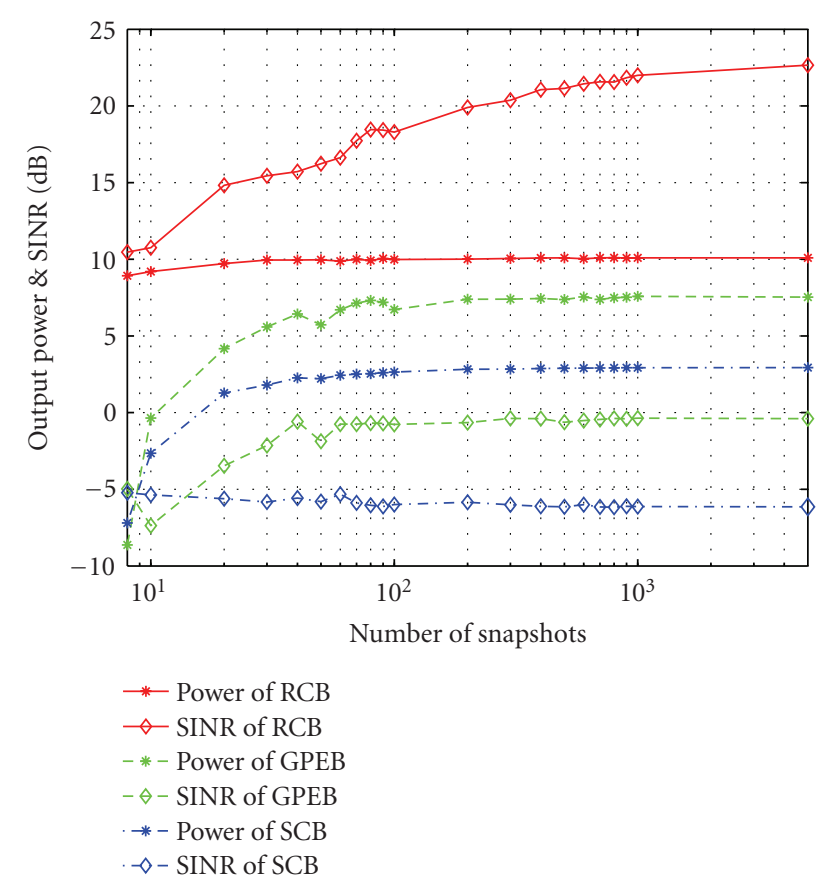

FIGURE 5: Output power and SINR versus the number of snapshots with random ASV error $(\epsilon=0.03)$.

with significantly higher accuracy than that of the SCB estimates.

From the simulation results shown in Figures 1 and 2, we find that the RCB cannot achieve the highest output SINR of the SCB with known ASV. Although the derived optimal output SINR $\rho_{u}$ in Lemma 2 is very close to the ideal output 


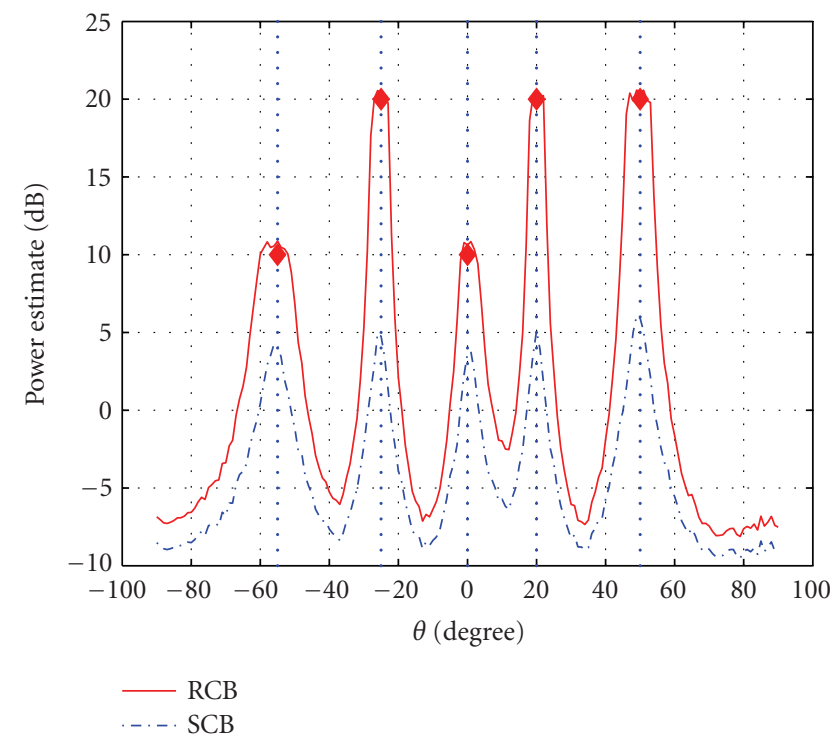

FIGURE 6: Comparison of power estimation of RCB and SCB versus steering direction. (The vertical dotted lines and the diamonds indicate the direction and the true power of each signal; $\epsilon=0.1$.)

SINR when the dimension of $\mathbf{U}_{n}$ is high, we point out in Section 3 that the RCB cannot achieve this optimal output SINR. The last experiment is carried out to compare the output SINR of the RCB with its bound. The output SINR of the SCB with known ASV is also evaluated. In the simulation, the steering direction error changes from $1^{\circ}$ to $10^{\circ}$. The results in Figure 7 show that the bound of the RCB is lower than the SINR of the SCB with known ASV. The output SINR of the $\mathrm{RCB}$ is close to its bound when the steering error is small. Although the output SINR of the RCB is lower than its bound, it still demonstrates high robustness to steering vector error as shown in all experiments.

\section{CONCLUSION}

The proposed robust beamforming method can be considered as maximizing the output power of the standard Capon beamformer. The derivation clearly shows the relationship between the proposed method and the beamforming method based on principal component analysis technique. Due to the existence of strong interference, uncertainty constraint is applied on the nominal array steering vector to prevent the RCB from target signal cancellation. Simulation results show that the proposed beamformer is robust to arbitrary array steering vector. The study on SINR improvement of the RCB also shows that the RCB does not achieve its optimal output SINR. Future work can be carried out to further improve its output SINR.

\section{APPENDIX}

\section{PROOF OF LEMMA 2}

The proposed RCB uses the ASV $\hat{\mathbf{s}}_{0}$ given in (10) instead of the nominal ASV $\overline{\mathbf{s}}_{0}$ in the calculation of optimal weight vector (11). Refer to Lemma 1; the bound of output SINR of

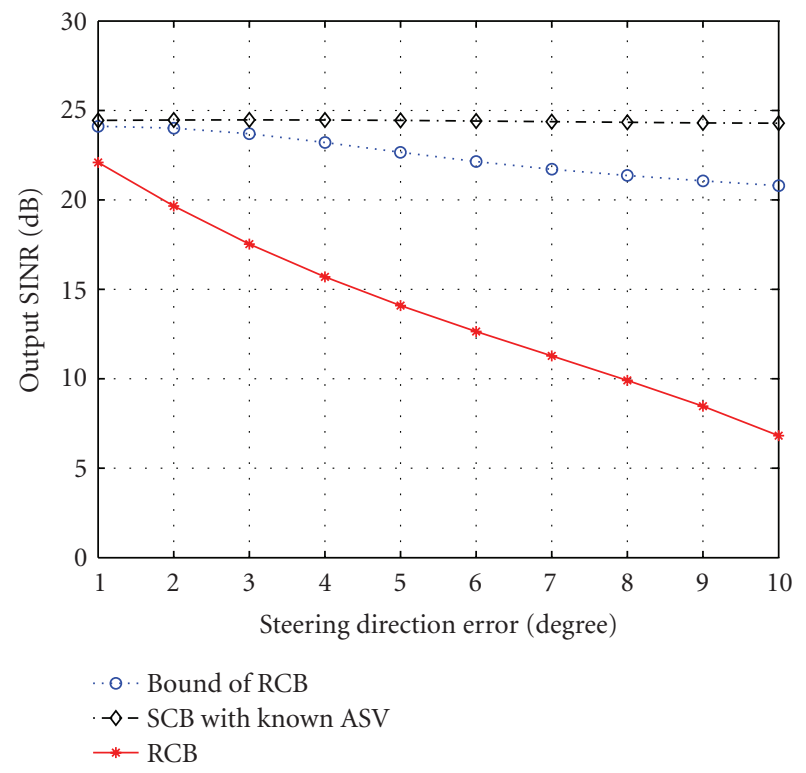

FIGURE 7: Comparison of output SINR of the RCB with its bound.

the proposed RCB can be obtained by studying the angle between the ASV $\hat{\mathbf{s}}_{0}$ and the true one $\mathbf{s}_{0}$. Another proof can be found in [23].

The array covariance matrix can be expressed as

$$
\mathbf{R}=\sigma_{s}^{2} \mathbf{s}_{0} \mathbf{s}_{0}^{H}+\mathbf{R}_{n}
$$

Using matrix inversion lemma, we have

$$
\mathbf{R}^{-1}=\mathbf{R}_{n}^{-1}-\frac{\sigma_{s}^{2}}{1+\xi}\left(\mathbf{R}_{n}^{-1} \mathbf{s}_{0}\right)\left(\mathbf{R}_{n}^{-1} \mathbf{s}_{0}\right)^{H}
$$

where $\xi=\sigma_{s}^{2} \mathbf{s}_{0}^{H} \mathbf{R}_{n}^{-1} \mathbf{s}_{0}$

Using matrix inversion lemma again,

$$
\left(\mathbf{R}^{-1}+g \mathbf{I}\right)^{-1}=\left(\mathbf{R}_{n}^{-1}+g \mathbf{I}\right)^{-1}+\frac{k\left(\mathbf{I}+g \mathbf{R}_{n}\right)^{-1} \mathbf{s}_{0} \mathbf{s}_{0}^{H}\left(\mathbf{I}+g \mathbf{R}_{n}\right)^{-1}}{1-k \mathbf{s}_{0}^{H}\left(\mathbf{R}_{n}+g \mathbf{R}_{n}^{2}\right)^{-1} \mathbf{s}_{0}},
$$

where $k=\sigma_{s}^{2} /(1+\xi)$.

Substituting (A.3) into (10), we have

$$
\begin{aligned}
\widehat{\mathbf{s}}_{0} & =\left(\mathbf{R}^{-1}+g_{1} \mathbf{I}\right)^{-1} \overline{\mathbf{s}}_{0} \\
& =\left(\mathbf{R}_{n}^{-1}+g_{1} \mathbf{I}\right)^{-1} \overline{\mathbf{s}}_{0}+d\left(\mathbf{I}+g_{1} \mathbf{R}_{n}\right)^{-1} \mathbf{s}_{0},
\end{aligned}
$$

where $d=k \mathbf{s}_{0}^{H}\left(\mathbf{I}+g_{1} \mathbf{R}_{n}\right)^{-1} \overline{\mathbf{s}}_{0} /\left(1-k \mathbf{s}_{0}^{H}\left(\mathbf{R}_{n}+g_{1} \mathbf{R}_{n}^{2}\right)^{-1} \mathbf{s}_{0}\right)$.

Assuming that the angle between $\hat{\mathbf{s}}_{0}$ and $\mathbf{s}_{0}$ is $\theta$, we have

$$
\cos ^{2}(\theta)=\frac{\left|\hat{\mathbf{s}}_{0}^{H} \mathbf{R}_{n}^{-1} \mathbf{s}_{0}\right|^{2}}{\left\|\mathbf{s}_{0}\right\|_{\mathbf{R}}^{2}\left\|\hat{\mathbf{s}}_{0}\right\|_{\mathbf{R}}^{2}}
$$


The items in (A.5) can be calculated as

$$
\begin{aligned}
\hat{\mathbf{s}}_{0}^{H} \mathbf{R}_{n}^{-1} \mathbf{s}_{0}= & \overline{\mathbf{s}}_{0}^{H}\left(\mathbf{I}+g_{1} \mathbf{R}_{n}\right)^{-1} \mathbf{s}_{0}+d^{*} \mathbf{s}_{0}^{H}\left(\mathbf{R}_{n}+g_{1} \mathbf{R}_{n}^{2}\right)^{-1} \mathbf{s}_{0}, \\
\left\|\hat{\mathbf{s}}_{0}\right\|_{\mathbf{R}}^{2}= & \widehat{\mathbf{s}}_{0}^{H} \mathbf{R}_{n}^{-1} \hat{\mathbf{s}}_{0} \\
= & \overline{\mathbf{s}}_{0}^{H}\left(\mathbf{I}+g_{1} \mathbf{R}_{n}\right)^{-2} \mathbf{R}_{n} \overline{\mathbf{s}}_{0}+2 \operatorname{Re}\left\{d \overline{\mathbf{s}}_{0}\left(\mathbf{I}+g_{1} \mathbf{R}_{n}\right)^{-2} \mathbf{s}_{0}\right\} \\
& +|d|^{2} \mathbf{s}_{0}^{H}\left(\mathbf{I}+g_{1} \mathbf{R}_{n}\right)^{-2} \mathbf{s}_{0},
\end{aligned}
$$

where $\operatorname{Re}\{\cdot\}$ is the real operator.

If we assume that the eigenvalues of $\Sigma_{i}$ are far greater than the variance of noise $\sigma_{n}^{2}$, using the eigendecomposition in (16), (A.6) can be approximated as

$$
\begin{aligned}
\hat{\mathbf{s}}_{0}^{H} \mathbf{R}_{n}^{-1} \mathbf{s}_{0}= & \overline{\mathbf{s}}_{0}^{H}\left(\mathbf{I}+g_{1} \mathbf{R}_{n}\right)^{-1} \mathbf{s}_{0}+d^{*} \mathbf{s}_{0}^{H}\left(\mathbf{R}_{n}+g_{1} \mathbf{R}_{n}^{2}\right)^{-1} \mathbf{s}_{0} \\
\approx & \frac{\overline{\mathbf{s}}_{0}^{H} \mathbf{U}_{n} \mathbf{U}_{n}^{H} \mathbf{s}_{0}}{1+\sigma_{n}^{2} g_{1}}+\frac{d^{*} \mathbf{s}_{0}^{H} \mathbf{U}_{n} \mathbf{U}_{n}^{H} \mathbf{s}_{0}}{\sigma_{n}^{2}\left(1+\sigma_{n}^{2} g_{1}\right)} \\
= & \frac{\psi_{c}}{1+\sigma_{n}^{2} g_{1}}+\frac{d^{*} \psi_{0}}{\sigma_{n}^{2}\left(1+\sigma_{n}^{2} g_{1}\right)}, \\
\left\|\hat{\mathbf{s}}_{0}\right\|_{\mathbf{R}}^{2}= & \overline{\mathbf{s}}_{0}^{H}\left(\mathbf{I}+g_{1} \mathbf{R}_{n}\right)^{-2} \mathbf{R}_{n} \overline{\mathbf{s}}_{0}+2 \operatorname{Re}\left\{d \overline{\mathbf{s}}_{0}\left(\mathbf{I}+g_{1} \mathbf{R}_{n}\right)^{-2} \mathbf{s}_{0}\right\} \\
& +|d|^{2} \mathbf{s}_{0}^{H}\left(\mathbf{I}+g_{1} \mathbf{R}_{n}\right)^{-2} \mathbf{s}_{0} \\
\approx & \frac{\sigma_{n}^{2} \overline{\mathbf{s}}_{0}^{H} \mathbf{U}_{n} \mathbf{U}_{n}^{H} \overline{\mathbf{s}}_{0}}{\left(1+g_{1} \sigma_{n}^{2}\right)^{2}}+2 \operatorname{Re}\left\{\frac{d \overline{\mathbf{s}}_{0} \mathbf{U}_{n} \mathbf{U}_{n}^{H} \mathbf{s}_{0}}{\left(1+g_{1} \sigma_{n}^{2}\right)^{2}}\right\} \\
& +\frac{|d|^{2} \mathbf{s}_{0}^{H} \mathbf{U}_{n} \mathbf{U}_{n}^{H} \mathbf{s}_{0}}{\left(1+g_{1} \sigma_{n}^{2}\right)^{2}} \\
= & \frac{\sigma_{n}^{2} \psi_{b}}{\left(1+g_{1} \sigma_{n}^{2}\right)^{2}}+2 \operatorname{Re}\left\{\frac{d \psi_{c}}{\left(1+g_{1} \sigma_{n}^{2}\right)^{2}}\right\}+\frac{|d|^{2} \psi_{0}}{\left(1+g_{1} \sigma_{n}^{2}\right)^{2}},
\end{aligned}
$$

where

$$
\psi_{c}=\overline{\mathbf{s}}_{0}^{H} \mathbf{U}_{n} \mathbf{U}_{n}^{H} \mathbf{s}_{0}, \quad \psi_{0}=\mathbf{s}_{0}^{H} \mathbf{U}_{n} \mathbf{U}_{n}^{H} \mathbf{s}_{0}, \quad \psi_{b}=\overline{\mathbf{s}}_{0}^{H} \mathbf{U}_{n} \mathbf{U}_{n}^{H} \overline{\mathbf{s}}_{0} .
$$

If the angle between $\widehat{\mathbf{s}}_{0}$ and $\mathbf{s}_{0}$ is $\theta$, we have

$$
\begin{aligned}
f & =\cos ^{2}(\theta)=\frac{\left|\hat{\mathbf{s}}^{H} \mathbf{R}_{n}^{-1} \mathbf{s}_{0}\right|^{2}}{\left\|\mathbf{s}_{0}\right\|_{\mathbf{R}}^{2}\|\hat{\mathbf{s}}\|_{\mathbf{R}}^{2}} \\
& =\frac{\left|\psi_{c}+\left(d^{*} \psi_{0} / \sigma_{n}^{2}\right)\right|^{2}}{\left\|\mathbf{s}_{0}\right\|_{\mathbf{R}}^{2}\left(\sigma_{n}^{2} \psi_{b}+2 \operatorname{Re}\left\{d \psi_{c}\right\}+\left(|d|^{2} \psi_{0} / \sigma_{n}^{2}\right)\right)} .
\end{aligned}
$$

Substitute

$$
\begin{aligned}
d & =\frac{k \mathbf{s}_{0}^{H}\left(\mathbf{I}+g_{1} \mathbf{R}_{n}\right)^{-1} \overline{\mathbf{s}}_{0}}{1-k \mathbf{s}_{0}^{H}\left(\mathbf{R}_{n}+g_{1} \mathbf{R}_{n}^{2}\right)^{-1} \mathbf{s}_{0}} \\
& \approx \frac{k \sigma_{n}^{2} \psi_{c}^{*}}{\sigma_{n}^{2}\left(1+g_{1} \sigma_{n}^{2}\right)-k \psi_{0}}=\frac{k \sigma_{n}^{2} \psi_{c}^{*}}{\beta},
\end{aligned}
$$

where $\beta=\sigma_{n}^{2}\left(1+g_{1} \sigma_{n}^{2}\right)-k \psi_{0}$. Substituting $d$ into (A.9), we have

$$
\begin{aligned}
f(\beta) & =\frac{\left|\psi_{c}+\left(d^{*} \psi_{0} / \sigma_{n}^{2}\right)\right|^{2}}{\left\|\mathbf{s}_{0}\right\|_{\mathbf{R}}^{2}\left(\sigma_{n}^{2} \psi_{b}+2 \operatorname{Re}\left\{d \psi_{c}\right\}+\left(|d|^{2} \psi_{0} / \sigma_{n}^{2}\right)\right)} \\
& =\frac{\left|\psi_{c}\right|^{2}\left(\beta+k \psi_{0}\right)^{2}}{\left\|\mathbf{s}_{0}\right\|_{\mathbf{R}}^{2}\left(\sigma_{n}^{2} \psi_{b} \beta^{2}+2 k \sigma_{n}^{2}\left|\psi_{c}\right|^{2} \beta+k^{2} \sigma_{n}^{2} \psi_{0}\left|\psi_{c}\right|^{2}\right)} .
\end{aligned}
$$

It is obvious that if $\left|\psi_{c}\right|^{2}=0$, then $\cos ^{2}(\theta) \equiv 0$. In such a case, the beamformer cannot work. The maximum value of $\cos ^{2}(\theta)$ is achieved when $d f(\beta) / d \beta=0$. After some straightforward algebraic manipulations, it yields

$$
\beta=0
$$

Hence,

$$
\sigma_{n}^{2}\left(1+g_{1} \sigma_{n}^{2}\right)-k \psi_{0}=0
$$

Therefore, the upper bound of the output SINR is achieved when the value of $g_{1}$ satisfies

$$
g_{1}=\frac{-1}{\sigma_{n}^{2}+\sigma_{s}^{2} \psi_{0}}=\frac{-1}{\sigma_{n}^{2}+\sigma_{s}^{2}\left\|P_{\mathbf{U}_{n}} \mathbf{s}_{0}\right\|^{2}},
$$

and the corresponding output SINR is

$$
\rho_{o}=\frac{\sigma_{s}^{2}\left\|P_{\mathbf{U}_{n}} \mathbf{s}_{0}\right\|^{2}}{\sigma_{n}^{2}} .
$$

\section{REFERENCES}

[1] J. Capon, "High-resolution frequency-wavenumber spectrum analysis," Proceedings of the IEEE, vol. 57, no. 8, pp. 1408-1418, 1969.

[2] L. C. Godara, "The effect of phase-shifter errors on the performance of an antenna-array beamformer," IEEE Journal of Oceanic Engineering, vol. 10, no. 3, pp. 278-284, 1985.

[3] J. W. Kim and C. K. Un, "An adaptive array robust to beam pointing error," IEEE Transactions on Signal Processing, vol. 40, no. 6, pp. 1582-1584, 1992.

[4] N. K. Jablon, "Adaptive beamforming with the generalized sidelobe canceller in the presence of array imperfection," IEEE Transactions on Antennas Propagation, vol. 34, no. 8, pp. 9961012, 1986.

[5] Y. J. Hong, C.-C. Yeh, and D. R. Ucci, "The effect of a finitedistance signal source on a far-field steering Applebaum arraytwo dimensional array case," IEEE Transactions on Antennas and Propagation, vol. 36, no. 4, pp. 468-475, 1988.

[6] S. Affes and Y. Grenier, "A signal subspace tracking algorithm for microphone array processing of speech," IEEE Transactions on Speech and Audio Processing, vol. 5, no. 5, pp. 425-437, 1997.

[7] J. E. Hudson, Adaptive Array Principles, Peter Peregrinus, London, UK, 1981.

[8] K. L. Bell, Y. Ephraim, and H. L. Van Trees, "A Bayesian approach to robust adaptive beamforming," IEEE Transactions on Signal Processing, vol. 48, no. 2, pp. 386-398, 2000. 
[9] A. B. Gershman, "Robust adaptive beamforming in sensor arrays," AEU-International Journal of Electronics and Communications, vol. 53, no. 6, pp. 305-314, 1999.

[10] M. H. Er and B. C. Ng, "A new approach to robust beamforming in the presence of steering vector errors," IEEE Transactions on Signal Processing, vol. 42, no. 7, pp. 1826-1829, 1994.

[11] Z. L. Yu, Q. Zou, and M. H. Er, "A new approach to robust beamforming against generalized phase errors," in Proceedings of the 6th IEEE Circuits and Systems Symposium on Emerging Technologies, vol. 2, pp. 775-778, Shanghai, China, May-June 2004.

[12] Q. Zou, Z. L. Yu, and Z. Lin, "A robust algorithm for linearly constrained adaptive beamforming," IEEE Signal Processing Letters, vol. 11, no. 1, pp. 26-29, 2004.

[13] M. S. Bazaraa and C. M. Shetty, Nonlinear Programming: Theory and Algorithms, John Wiley \& Sons, New York, NY, USA, 1979.

[14] I. T. Jolliffe, Principal Component Analysis, Springer, New York, NY, USA, 1986.

[15] S. A. Vorobyov, A. B. Gershman, and Z.-Q. Luo, "Robust adaptive beamforming using worst-case performance optimization: a solution to the signal mismatch problem," IEEE Transactions on Signal Processing, vol. 51, no. 2, pp. 313-324, 2003.

[16] P. Stoica, Z. Wang, and J. Li, "Robust Capon beamforming," IEEE Signal Processing Letters, vol. 10, no. 6, pp. 172-175, 2003.

[17] J. Li, P. Stoica, and Z. Wang, "On robust Capon beamforming and diagonal loading," IEEE Transactions on Signal Processing, vol. 51, no. 7, pp. 1702-1715, 2003.

[18] J. Li, P. Stoica, and Z. Wang, "Doubly constrained robust Capon beamformer," IEEE Transactions on Signal Processing, vol. 52, no. 9, pp. 2407-2423, 2004.

[19] H. Cox, "Resolving power and sensitivity to mismatch of optimum array processors," The Journal of the Acoustical Society of America, vol. 54, no. 3, pp. 771-785, 1973.

[20] R. T. Compton, "The effect of random steering vector errors in the Applebaum adaptive array," IEEE Transactions on Aerospace and Electronic Systems, vol. 18, no. 5, pp. 392-400, 1982.

[21] B. D. Van Veen and R. A. Roberts, "Partially adaptive beamformer design via output power minimization," IEEE Transactions on Acoustics, Speech, and Signal Processing, vol. 35, no. 11, pp. 1524-1532, 1987.

[22] B. D. Van Veen, "An analysis of several partially adaptive beamformer designs," IEEE Transactions on Acoustics, Speech, and Signal Processing, vol. 37, no. 2, pp. 192-203, 1989.

[23] F. Vincent and O. Besson, "Steering vector errors and diagonal loading," IEE Proceedings of Radar, Sonar and Navigation, vol. 151, no. 6, pp. 337-343, 2004.

Zhu Liang YU received his BSEE degree in 1995 and MSEE degree in 1998, both in electronic engineering, from the Nanjing University of Aeronautics and Astronautics, China. He worked in Shanghai BELL Co. Ltd. as a Software Engineer from 1998 to 2000 . He joined Center for Signal Processing, Nanyang Technological University, from 2000, as a Research Engineer. Currently he is a Ph.D. candidate in School of

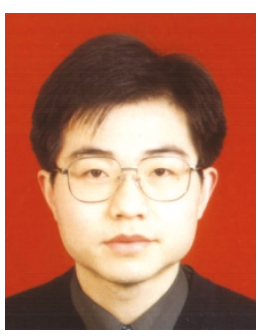
Electrical and Electronic Engineering, Nanyang Technological University, Singapore. His research interests include array signal processing, acoustic signal processing, and adaptive signal processing.
Meng Hwa Er received the B. Eng. degree in electrical engineering with 1st class honors from the National University of Singapore in 1981, and the Ph.D. degree in electrical and computer engineering from the University of Newcastle, Australia, in 1986. He joined the Nanyang Technological Institute/University in 1985 and was promoted to a Full Professor in 1996. He served as an Associate Editor of the IEEE Transactions

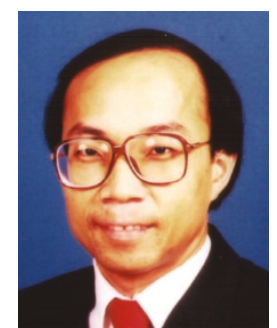
on Signal Processing from 1997 to 1998 and is a Member of the Editorial Board of IEEE Signal Processing Magazine from 2005 to 2007. He was the General Cochair of the IEEE International Conference on Image Processing, 2004. His research interests include array signal processing, satellite communications, computer vision, and optimization techniques. 